\title{
Probing Mechanisms of Photochemical Reactions: How to Teach a Young Dog Old Tricks
}

\author{
Christian G. Bochet
}

\begin{abstract}
The mechanism of photochemical reactions can be difficult to study because of the very short-lived intermediates involved. State-of-the art ultrafast spectroscopic tools can be used to probe these processes, but we will show in this account that old-fashioned techniques, such as the determination of quantum yields, the measurement of isotope effects or the use of triplet quenchers and sensitizers still can give a significant insight into the mechanism of photochemical reactions.
\end{abstract}

Keywords: Isotope effect - Mechanisms · Photochemistry · Quantum yield · Triplet reaction

\section{Introduction}

There is no need to detail here why understanding reaction mechanisms is important, and many articles of this special issue address this problem. In photochemical reactions, this is of particular importance, because these reactions often follow multiple parallel pathways (some of them leading to the same observed product), and because they are notoriously difficult to troubleshoot. In a thermal reaction (in this article, we will use this word not to suggest the use of particular heating, but rather meaning 'non-photochemical'), the reactant is converted into the product following the reaction coordinate through an energy saddle point: the transition state. A typical way of influencing the course of a reaction is to change some of the reaction parameters (temperature, solvents, concentration) moving up or down the energy levels of the reactant, the product or the transition state.

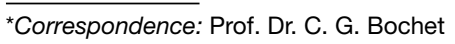
Department of Chemistry, University of Fribourg Ch. du Musée 9, CH-1700 Fribourg, Switzerland E-mail: christian.bochet@unifr.ch
For example, adjusting the medium in order to destabilize the reactant and stabilize the transition state will result in an acceleration of the process. In some photochemical reactions, a very similar scenario can occur, this time on an electronically excited potential energy surface: for example the reactant is excited into its $S_{1}$ state, where it meets a new surface with a barrier hopefully capable of being overcome at room temperature, thus leading to the product, still in its $S_{1}$ state. Fluorescence or non-radiative deactivation gives then the expected product. Unfortunately, such reactions, called adiabatic, belong to a minority. Indeed, the $S_{1}$ surface usually intersects the ground-state surface $S_{0}$ somewhere, even if it is in a region far away from the reaction coordinate. Such a crossing is called conical intersection, and it represents an efficient relaxation pathway towards the ground state. Depending on the location of this intersection, and the dynamics of the molecule reaching an intersection, the excited molecule may return to the reactant side, or end up as the product. Whether the crossing is allowed, avoided or forbidden is beyond the scope of this article, but their existence is a fact, and many examples of them have been studied computationally. In fact, different excited surfaces also cross each other, which leads to multiple conical intersections, the location of which can be dramatically affected by even small influences, e.g. the presence or absence of a given substituent at a given position, the solvent, the $\mathrm{pH}$ or coordination with metal ions. This extreme sensitivity and complexity is certainly one of the reasons why photochemistry is often considered unpredictable, thus making its understanding even more important. Fortunately, there is a substantial toolbox at the disposition of the experimental photochemist, and we will discuss here some of them, illustrated by examples from our own work.

\section{Time-resolved Spectroscopy}

Time-resolved spectroscopy is a technique allowing the measurement of a molecular spectrum at a well-defined instant after triggering an event. One of the most widely used of these techniques is the measurement of a UV/Vis spectrum a few moments after the excitation of a molecule by a flash of light (hence the popular term flash photolysis). Typical time scales can be from a few femtoseconds (requiring very costly laser setups) to a few microseconds. Comparison of the absorption or emission with varying delays between the flash and the measurement of the spectra allows for an accurate determination of the excited-state lifetime. It is undeniable that flash photolysis has allowed complex problems in chemistry to be solved; its value has actually been recognized by awarding the Nobel Prize in chemistry to George Porter in 1967. On the other hand, while the time component of such experiments is very reliable, there is the temptation to over-interpret the transient UV/Vis spectra and to hypothesize structures. After all, no sensible peer reviewer would accept a single UV spectrum as proof of identity for a newly synthesized organic compound. There are however other well-established time-resolved methods, such as FT-IR and $\mathrm{X}$-ray. The latter is of course of extreme usefulness, but its demanding experimental setup bans it for the moment from the organic chemist's laboratory (it requires a synchrotron!). Thus, in our opinion, FT-IR remains the most valuable variant, as, in 
spite of not being able to confirm the whole structure, it can confirm the presence or absence of particular functional groups. As an example, the photoacylation ${ }^{[1]}$ of amine nucleophiles by $N$-acetyldinitroindolines 1 was studied by this technique (Scheme 1).

Different mechanisms could in principle be envisaged, such as direct trapping of the excited state by the nucleophile, a radical pathway or the photochemical formation of an intermediate, later converted into the product in a bimolecular process. In collaboration with the group of John Toscano at Johns Hopkins University, we followed the process by time-resolved FT-IR. ${ }^{22]}$ Immediately after photolysis at $355 \mathrm{~nm}$, a new product is visible, decomposing then with a typical half-life of a few dozen microseconds (Scheme 1a). A characteristic vibration at around $1800 \mathrm{~cm}^{-1}$ was compatible with a very electrophilic carbonyl compound, such as $\mathbf{2}$ (Scheme 1b). DFT calculation of the full vibrational spectrum further confirmed this assignment. There are actually two isomers of 2 visible: one of them returns back to the ground state of $\mathbf{1}$, whereas the other reacts with the nucleophile (here butylamine) leading to the amide 3 .

\section{Quantum Yields}

In a thermal reaction, one of the most important descriptors of the process is the yield, which reflects the proportion of the reactant molecules ending up as product. In addition to the practical or economical value, this indicates which of the multiple possible paths on the potential energy surface is the most likely to be followed (and we assume here an infinitely skilled experimentalist). The same applies to photochemical reactions, where one considers photons as a reagent. Thus, the quantum yield for a particular process is defined as the proportion of effectively absorbed photons leading to products. As in its thermal counterpart, the quantum yield depicts how likely the pathway leading to the product is followed; as we have seen earlier, the number or the position of conical intersections has a bearing on the probability of obtaining the product, as well as other phenomena such as intersystem crossing and internal conversion. Thus the quantum yield is a very useful tool to study a reaction mechanism.

Its measurement is, however, less straightforward than a simple yield determination, as the photon stoichiometry needs to be known. By making sure that practically all the photons emitted by the lamp in the direction of the sample are absorbed (i.e. by adjusting the geometrical factors of the experimental setup and by ensuring an optical density larger than 2), one can re-
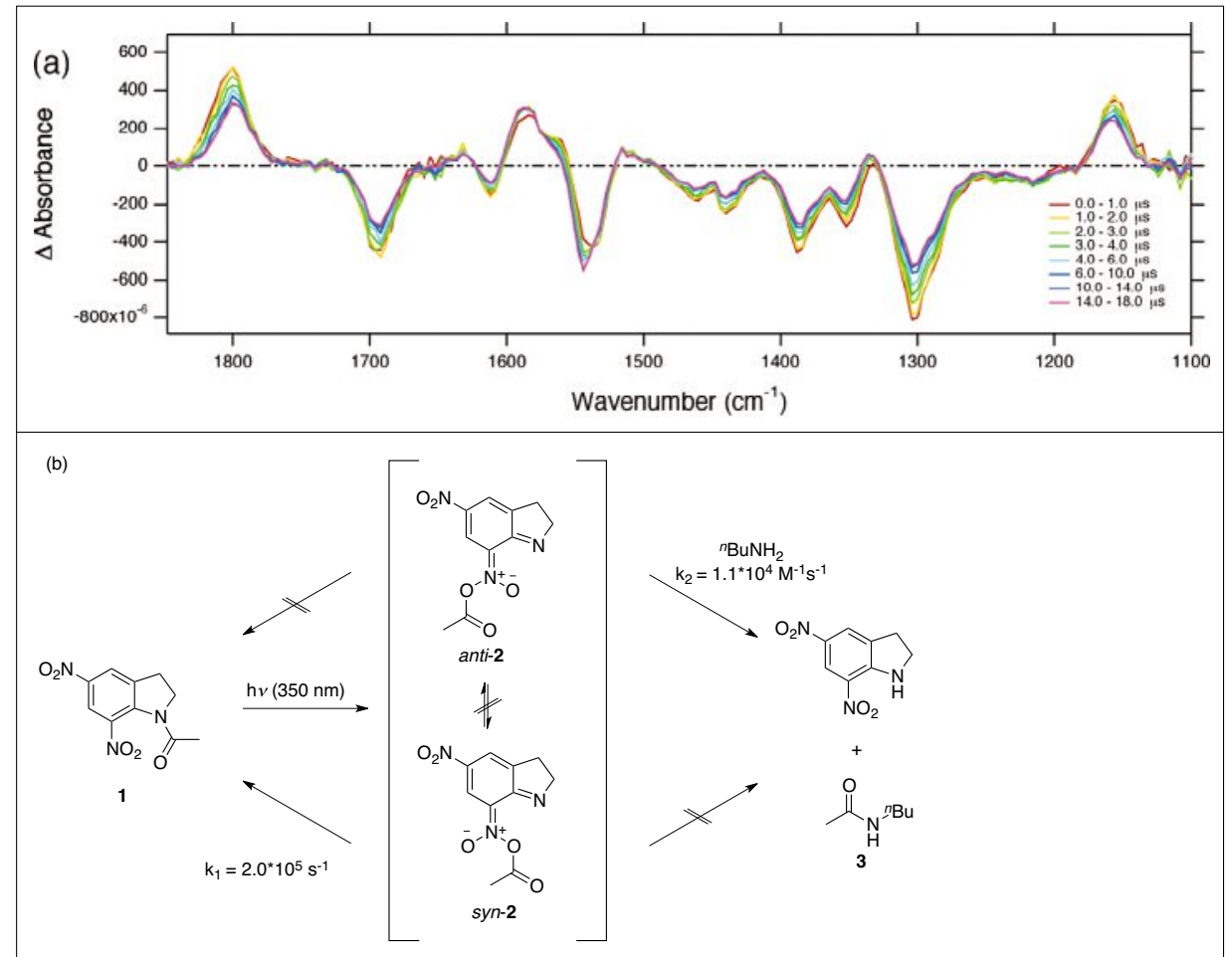

Scheme 1. Study of a photoacylation reaction by TR-FTIR. Adapted with permission from ref. [2]. Copyright 2005 American Chemical Society.

duce the problem to knowing the number of photons emitted by the light source. This could in principle be determined by using suitable physical instruments, but these are often either impractical, unreliable or too wavelength-dependent to be useful in a typical preparative laboratory. An alternative is the measurement of the chemical yield of a standard photochemical reaction, of which the quantum yield is known, under the exact same experimental conditions as the new reaction (including the wavelength, of which the quantum yield can be dependent). [3] Photoreduction of potassium ferrioxalate, ${ }^{[4]}$ decomposition of phenyl glyoxylic acid[5] or fragmentation of valerophenone ${ }^{[6]}$ are very popular examples of such chemical actinometers. In our own laboratory, we tend to favor the two former reactions, with a preference to phenylglyoxylic decarboxylation (because quantifying either the initial phenyl glyoxylic acid or the resulting benzaldehyde is very simple); on the other hand, ferrioxalate actinometry is very precise, but it involves titration of $\mathrm{Fe}$ (II), which is not particulary popular among graduate students. The quantum yield $\Phi$ is a useful parameter to describe the efficiency of a photochemical process, irrespective of the experimental setup (i.e. geometry or intensity of the light source) and the absorbance (in some cases, the overall observed efficiency may depend on the absorbance; then, the $\varepsilon \Phi$ product is used). In the following example (Scheme 2), the quantum yield of photolysis of derivatives of $\mathbf{4}$ as a function of the leaving group $\mathrm{X}^{-}$was determined. [7] This has some importance in the context of the photoliberation of biologically relevant molecules by irradiation with light ('photouncaging'), as ortho-nitrobenzylic derivatives are the most popular photocages used currently. Scheme 2 summarizes the values of $\Phi$, and it becomes immediately apparent that the nature of the leaving group has a strong impact on the quantum yield. As $\Phi$ is setup- and absorbance-independent, the cause for this disparity has to be found in the photolysis mechanism itself. We refer the reader to the original articles for a deeper discussion, but, in short, a correlation between $\Phi$ and the radical stabilization energy of $\mathrm{X}$ was found, a fact that properly explains the counterintuitive higher reactivity for the poorest nucleofugacity of the leaving group.

It should also be noted that, even in the case of inaccuracies in the determination of the light intensity (actinometry), as all the quantum yields were measured under identical experimental conditions, the relative values remain correct and the mechanistic reasoning is not impacted.

\section{Isotope Effects}

The observation of strong (H/D) primary isotope effects is a strong evidence for a X-H bond-breaking event as the rate-determining step. The same applies to photochemical reactions. For example, as shown in Scheme 3, in the Norrish-type II related 


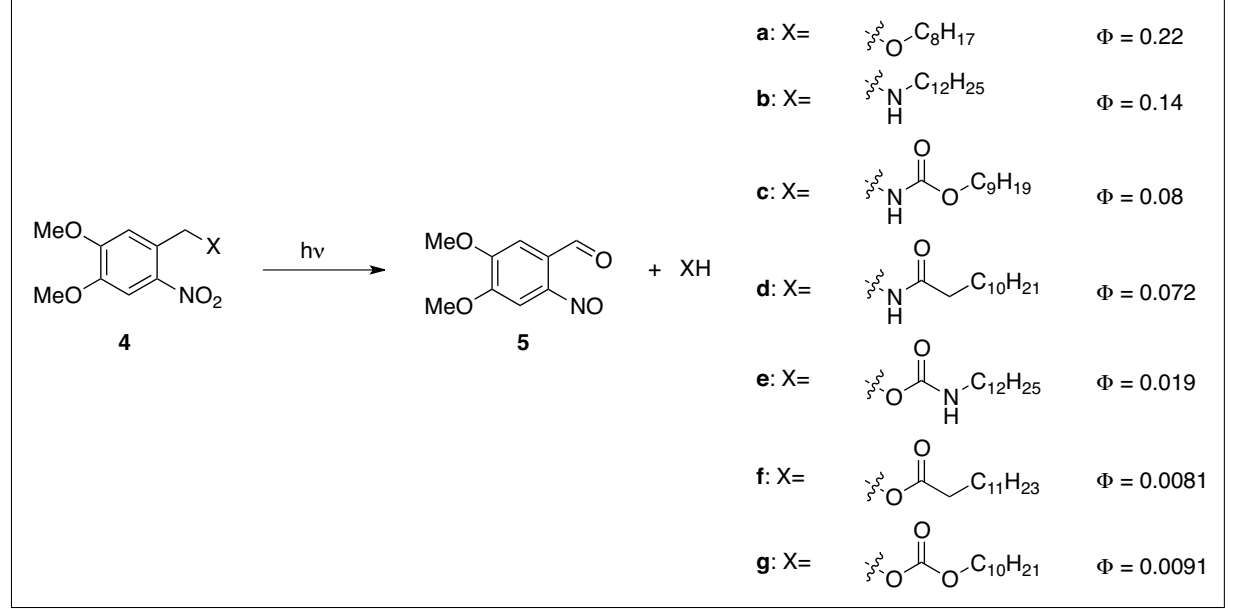

Scheme 2. Substituent effects on quantum yields.

photolysis of ortho-nitrobenzyl alcohol derivatives 6/7 (an extremely widespread photolabile protecting group, as mentioned earlier), it is quite obvious that a $\mathrm{C}-\mathrm{H}$ bond rupture is involved, but when it occurs is much less clear. ${ }^{[8]}$ In the following experiment that we conducted, by design, we did not probe the rate-determining step, but rather the product-determining step (i.e. an irreversible step leading to the product) A very significant excess of the $\mathbf{8}$ ester over its counterpart 9 is serious evidence that the $\mathrm{C}-\mathrm{H}$ bond rupture of $\mathbf{6}$ occurs much more rapidly than the $\mathrm{C}-\mathrm{D}$ rupture in 7 , and that this cleavage occurs at the product-determining step. This was actually exploited later for synthetic purposes, as playing with the type of isotope allowed a direct handle on the reaction rate. [9]

\section{Spin State}

The spin states involved in a photochemical reaction (either singlet or triplet in typical photochemical organic reactions) are probed mainly by the use of quenchers or sensitizers. A reaction accelerated by the use of a triplet sensitizer, which is irradiated at wavelengths where the substrate does not absorb, or a reaction suppressed by a triplet quencher is a reasonable indication of a triplet-based pathway. However, in the presence of a triplet quencher, a reaction may be forced to proceed through singlet excited states and is therefore not completely suppressed. In the following example (Scheme 4), the ortho-quinodimethane $\mathbf{1 1}$ is generated photochemically from 10, and reacts readily with dienophiles in a Diels-Alder reaction. While the latter reaction is typically stereospecific, the trans product $\mathbf{1 4}$ (relative configuration of both esters) is obtained as the major product, irrespective of the configuration of the dienophile (12 or 13), as shown in Table 1, entries 1 and 2 . This observation was made several decades ago, ${ }^{[10]}$ and the in situ photochemical isomerization of the alkene under the reaction conditions was invoked.[11]

Table 1. Effect of a quencher on the diastereospecificity.

${ }^{a}$ Yields estimated by ${ }^{1} \mathrm{H}-\mathrm{NMR}$

Table 2. Photoequilibration of diesters 12/13
However, when we probed this claim by irradiating dimethyl fumarate $\mathbf{1 2}$ and dimethyl maleate $\mathbf{1 3}$ separately under very similar reaction conditions $(350 \mathrm{~nm}$, acetonitrile, $4 \mathrm{~h}$ ), no significant isomerization was observed (Table 2, entries 1 and 2). ${ }^{[12]}$ There was, however, a missing component in the mixture: the aromatic aldehyde itself. Adding 0.5 equiv. benzaldehyde (a closely related analogue, which cannot form an ortho-quinodimethane), led to a photostationary equilibrium (Table 2 , entries 3 and 4). We hypothesized that benzaldehyde acted as a triplet sensitizer, and we attempted to suppress this pathway by adding a triplet quencher. Indeed, in the presence of the widely used piperylene (1,3-pentadiene; 0.5 equiv.), an efficient triplet quencher, we could, to a certain extent, restore the stereospecificity of the process (Table 1 , entry 3 ). In addition to the mechanistic interest, it is worth noting that a significant degree of molecular complexity is obtained in a single step start-

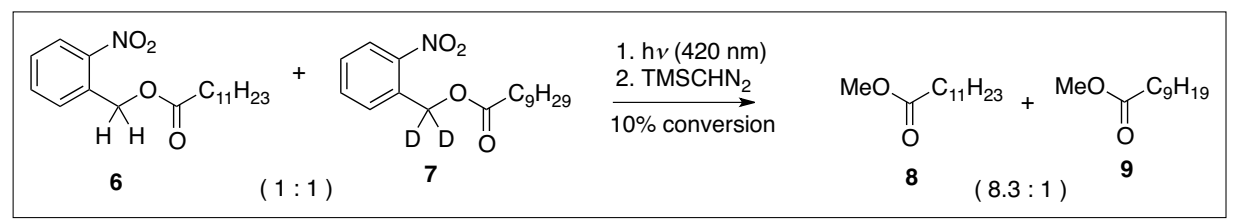

Scheme 3. Example of an isotope effect in a photochemical reaction.

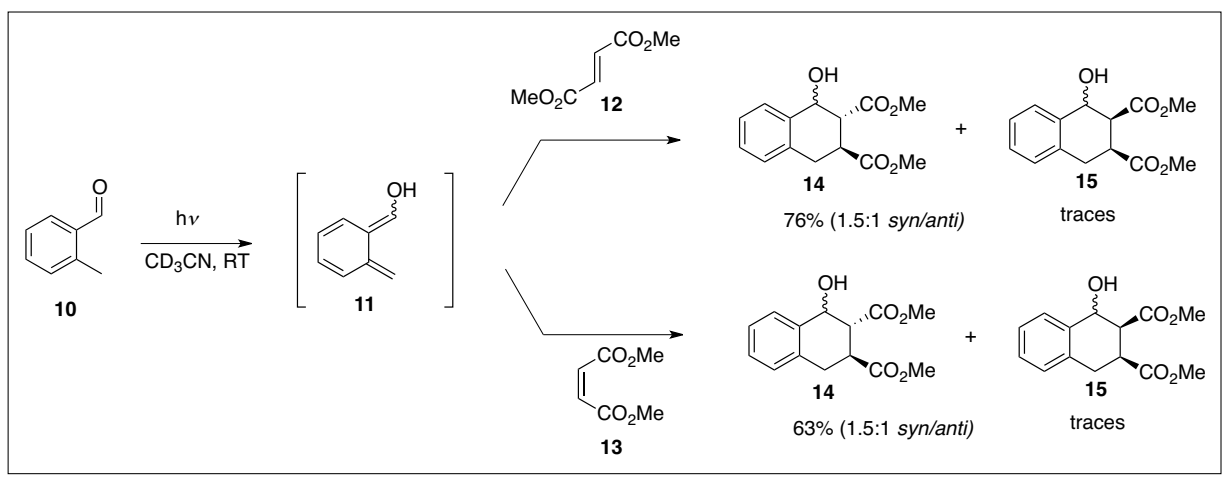

Scheme 4. Diastereospecificity in a photo-induced Diels-Alder reaction.

\begin{tabular}{|c|c|c|c|c|}
\hline entry & dienophile & additive & $\mathbf{1 4}($ syn/anti) & $\mathbf{1 5}($ syn/anti) \\
\hline 1 & $\mathbf{1 2}$ & - & $>99 \%(1.5: 1)$ & $<1 \%$ \\
\hline 2 & $\mathbf{1 3}$ & - & $92 \%(1.5: 1)$ & $8 \%(1: 1.7)$ \\
\hline 3 & $\mathbf{1 3}$ & piperylene & $28 \%(1.8: 1)$ & $72 \%(1.3: 1)$ \\
\hline
\end{tabular}

\begin{tabular}{|c|c|c|c|c|}
\hline entry & substrate & additive & $\mathbf{1 2}$ & $\mathbf{1 3}$ \\
\hline 1 & $\mathbf{1 2}$ & - & $97 \%$ & $3 \%$ \\
\hline 2 & $\mathbf{1 3}$ & - & $1 \%$ & $99 \%$ \\
\hline 3 & $\mathbf{1 2}$ & $\mathrm{PhCHO}$ & $12 \%$ & $88 \%$ \\
\hline 4 & $\mathbf{1 3}$ & $\mathrm{PhCHO}$ & $12 \%$ & $88 \%$ \\
\hline
\end{tabular}


ing with very inexpensive substrates, and that the relative configuration of two out of three stereocenters can now be controlled by the choice of the experimental conditions.

\section{Concluding Remarks}

These few modest examples show that relatively simple and quite old experimental techniques can provide useful insight into photochemical reactions, a notoriously difficult category of reactions to study. The use of more recent sophisticated spectroscopic techniques of course can further refine the mechanistic hypotheses obtained by these classical means, and both approaches should be used in a complementary fashion. In comparison with their ther- mal counterpart, photochemical reactions have been much less explored, and there is little doubt that a deeper understanding of their mechanisms, together with their use as key steps in the synthesis of complex molecules will gradually dissipate the common misconception of unpredictability and bring back essentially atomeconomical processes into the limelight.

Received: December 31, 2015

[1] B. Amit, D. A. Ben-Efraim, A. Patchornik, $J$. Am. Chem. Soc. 1976, 98, 843.

[2] A. D. Cohen, C. Helgen, C. G. Bochet, J. P. Toscano, Org. Lett. 2005, 7, 2845.

[3] For an excellent overview of the existing techniques, see: P. Klán, J. Wirz, 'Photochemistry of Organic Compounds: From Concepts to Practice', Wiley, Chichester, 2009.

[4] C. G. Hatchard, C. A. Parker, Proc. Roy. Soc. A: Math. Phys. Eng. Sci. 1956, 235, 518.
[5] H. J. Kuhn, H. Goerner, J. Phys. Chem. 1988, 92, 6208.

[6] R. G. Zepp, M. M. Guz, W. L. Miller, H. Gao, J. Phys. Chem. A 1998, 102, 5716.

[7] a) T. Šolomek, C. G. Bochet, T. Bally, Chem. Eur. J. 2014, 20, 8062; b) T. Šolomek, S. Mercier, T. Bally, C. G. Bochet, Photochem. Photobiol. Sci. 2012, 11, 548 .

[8] A. Blanc, C. G. Bochet, J. Am. Chem. Soc. 2004, 126, 7174

[9] A. Blanc, C. G. Bochet, Org. Lett. 2007, 9, 2649.

[10] T. Durst, E. C. Kozma, J. L. Charlton, J. Org. Chem. 1985, 50, 4829 .

[11] G. S. Hammond, J. Saltiel, A. A. Lamola, N. J. Turro, J. S. Bradshaw, D. O Cowan, R. C. Counsell, V. Vogt, C. Dalton, J. Am. Chem. Soc 1964, 86, 3197.

[12] a) J. Lage Robles, C. G. Bochet, unpublished results; b) J. Lage Robles, Thesis No 1570, University of Fribourg, 2007. 